\title{
SFS_CODE: More Efficient and Flexible Forward Simulations
}

\author{
Ryan D. Hernandez ${ }^{1,}$ and Lawrence H. Uricchio ${ }^{1,2}$ \\ 1Department of Bioengineering and Therapeutic Sciences; Institute for Human Genetics; Institute for Quantitative \\ Biosciences; University of California San Francisco, CA 94158. \\ ${ }^{2}$ Current address: Department of Biology; Stanford University, CA 94305.
}

\begin{abstract}
SUMMARY: Modern implementations of forward population genetic simulations are efficient and flexible, enabling the exploration of complex models that may otherwise be intractable. Here we describe an updated version of SFS_CODE, which has increased efficiency and includes many novel features. Among these features is an arbitrary model of dominance, the ability to simulate partial and soft selective sweeps, as well as track the trajectories of mutations and/or ancestries across multiple populations under complex models that are not possible under a coalescent framework. We also release sfs_coder, a Python wrapper to SFS_CODE allowing the user to easily generate command lines for common models of demography, selection, and human genome structure, as well as parse and simulate phenotypes from SFS_CODE output.
\end{abstract}

Availability and Implementation: Our open source software is written in C and Python, and are available under the GNU General Public License at http://sfscode.sourceforge.net.

Contact: ryan.hernandez@ucsf.edu

Supplementary information: Detailed usage information is available from the project website at http://sfscode.sourceforge.net.

\section{INTRODUCTION AND FEATURES}

Efficient, highly flexible forward genetic simulations are essential components of several lines of research. In population and evolutionary genetics, they are necessary to evaluate the robustness of theoretical models (Bank et al. 2014); in association studies, they are crucial for measuring statistical power (Uricchio et al. 2015); and future applications may include statistical inference (Buzbas \& Rosenberg 2015).

SFS_CODE is a computationally efficient implementation of a population genetic forward simulation. It has been used to understand the evolutionary forces driving patterns of genetic variation across many species (e.g. humans, Drosophila, plants, and coral), characterize complex demographic models, jointly model the effect of positive and negative selection, as well as designing statistical methods and sequencing studies. Since its introduction, SFS_CODE has undergone extensive revisions, and continues to be among the most efficient and flexible simulators available. Table 1 highlights novel features. In particular, efficient conditional simulations are now possible (e.g., using--trackTrajectory to retain simulations with specific terminal conditions, or --mutation to follow a specified trajectory).

We also introduced sfs_coder, a Python-based SFS_CODE interface that easily generates command-lines for several models of human demography, selection, and genome structure, as well as parses and analyzes SFS CODE output, simulates selection-based phenotypes, and provides rescaling methods for simulations of linked positive selection.

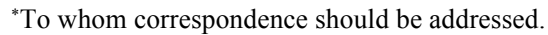


Table 1. Key novel features added to SFS CODE

\begin{tabular}{ll}
\hline Option & Description \\
\hline --sampSize & $\begin{array}{l}\text { Sample populations at defined } \\
\text { times } \\
\text { Dominance, selection on stand- } \\
\text { ing variation } \\
\text {--selDistType }\end{array}$ \\
$\begin{array}{l}\text { Locus-specific mutation rates } \\
\text { Join multiple populations at spec- } \\
\text { ified time }\end{array}$ \\
--rateClassLoci & $\begin{array}{l}\text { Report ancestry of each locus } \\
\text {--admix }\end{array}$ \\
Follow frequency trajectory of an \\
--trackAncestry \\
--trackTrajectory \\
Introduce mutations; follow \\
specified trajectory
\end{tabular}

See documentation for details

\section{METHODS}

Efficiency gains in SFS CODE have been achieved by storing all unique haplotypes in a locus as a collection of mutations in a randomized splay tree (a binary search tree that self-organizes upon accessing an element with probability $p$; (Albers \& Karpinski 2002)). Individuals in a population are then stored as arrays of haplotypes across loci. Since many haplotypes are introduced and lost each generation, SFS_CODE efficiently reuses lost haplotypes to reduce allocation and deallocation overhead.

While highly efficient for many use cases, these data structures can come at a cost when the number of unique haplotypes is large relative to the population size (e.g., long locus length or high mutation/recombination rate). SFS_CODE therefore benefits if long chromosomes are partitioned into smaller linked segments. The ideal partitioning depends on several parameters, and can be optimized heuristically with simulations that have a short burn-in time using the Perl script optimizeLL.pl now included in the distribution.

\subsection{Comparing performance}

We compared the run time of simulations using SFS_CODE to several other efficient forward simulation programs that have recently been released: FFPopSim (Zanini \& Neher 2012), fwdpp_ind (Thornton 2014), and SLiM (Messer 2013). Figure 1 shows the results, with the coalescent simulator ms (Hudson 2002) as a reference (which are often below scale as plotted). We varied the population size $(N)$ and total locus length $(L)$ for two values of the mutation $(\theta)$ and recombination $(\rho)$ rates (roughly corresponding to human and Drosophila parameters). We find that for short loci, FFPopSim can be extremely efficient, but it does not scale well with $L$. SFS_CODE (and the locus-optimized version) is often the most efficient. For large $L$ with unrealistically small $N$, forward simulations can be faster than the coalescent simulator $\mathrm{ms}$ (which has exponential runtime in $L$ with recombination; though see (Marjoram \& Wall 2006)). All simulations were run on an Intel Xeon 2.66GHz CPU.

\section{ACKNOWLEDGEMENTS}

Funding: This work was supported by a grant from the National Institutes of Health (1R01HG007644) and a Sloan Foundation Research Fellowship to RDH. 
SFS_CODE: More Efficient and Flexible Forward Simulations

\section{REFERENCES}

Albers S, Karpinski M (2002) Randomized splay trees: theoretical and experimental results. Information Processing Letters, 81, 213-221.

Bank C, Ewing GB, Ferrer-Admettla A, Foll M, Jensen JD (2014) Thinking too positive? Revisiting current methods of population genetic selection inference. Trends Genet, 30, 540-546.

Buzbas EO, Rosenberg NA (2015) AABC: approximate approximate Bayesian computation for inference in population-genetic models. Theor Popul Biol, 99, 31-42.

Hudson RR (2002) Generating samples under a Wright-Fisher neutral model of genetic variation. Bioinformatics, 18, 337-338.

Marjoram P, Wall JD (2006) Fast "coalescent" simulation. BMC Genet, 7, 16.

Messer PW (2013) SLiM: simulating evolution with selection and linkage. Genetics, 194, 1037-1039.

Thornton KR (2014) A C++ template library for efficient forward-time population genetic simulation of large populations. Genetics, 198, 157-166.

Uricchio LH, Torres R, Witte JS, Hernandez RD (2015) Population genetic simulations of complex phenotypes with implications for rare variant association tests. Genet Epidemiol, 39, 35-44.

Zanini F, Neher RA (2012) FFPopSim: an efficient forward simulation package for the evolution of large populations. Bioinformatics, 28, 3332-3333.
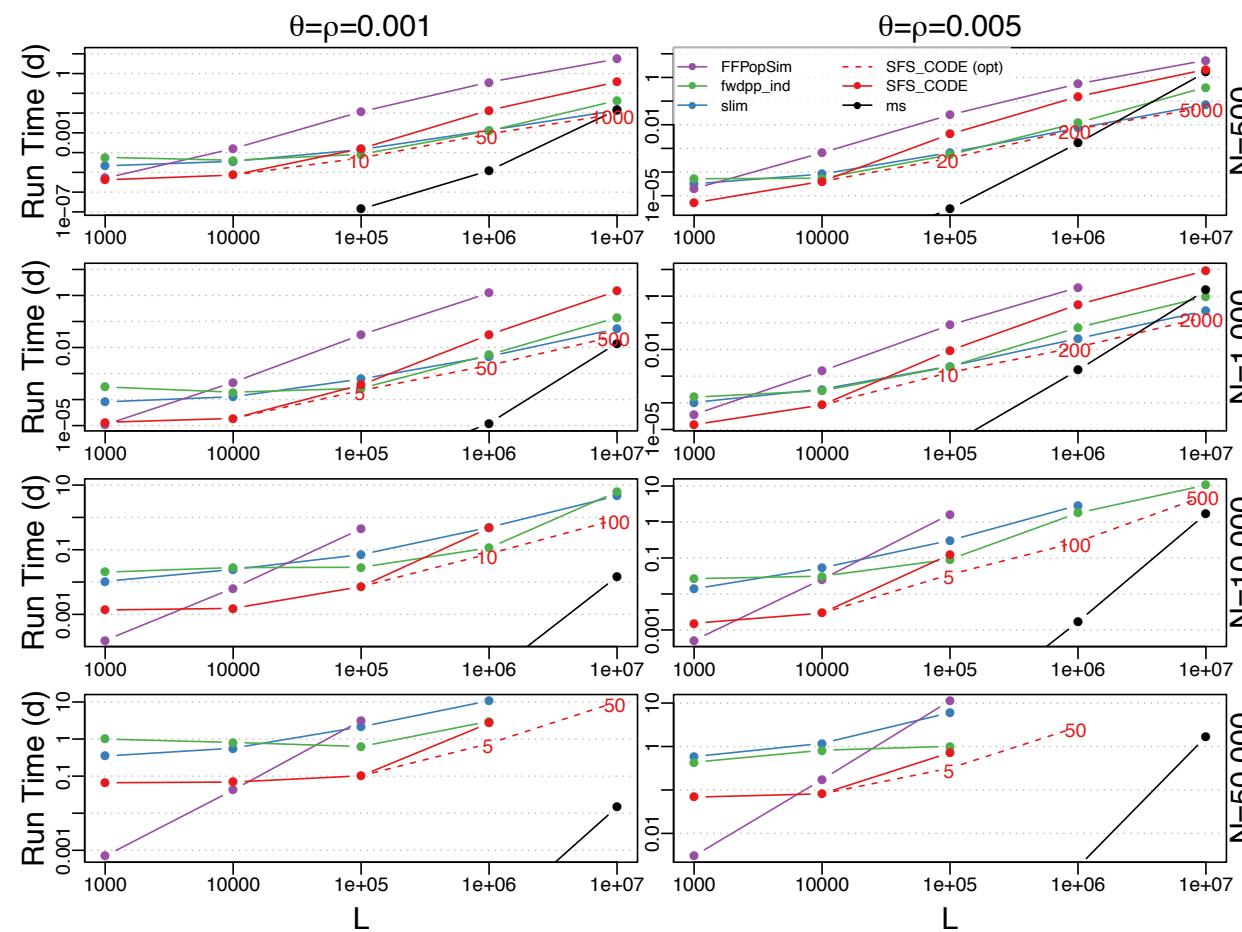
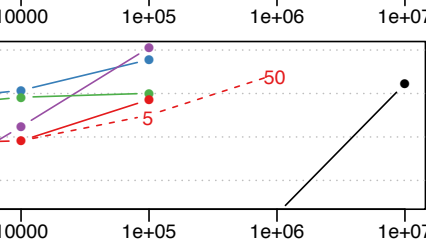

$\mathrm{L}$
Fig. 1. Mean run times 8 across simulators, representing 12.55 CPU years of computation (10 iterations per point). Each panel is a function of simulated sequence length, with lower II (human; left) and higher (Drosophila; right) mutation $(\theta)$ and recombination $(\rho)$ rates. The coalescent simulator ms is included in black - for reference. Dashed red II lines are a locus-optimized version of SFS_CODE, with the optimal number of loci 8 indicated in graph. Missing i points represent parameter II combinations that did not complete within 14 days. 